Revue d'histoire de l'Amérique française

REYUE D.HISTOIRE DE L'AMÉRIQUE FRANÇAISE

\title{
OUELLET, Danielle, avec la collaboration de René BUREAU, Franco Rasetti, physicien et naturaliste. Il a dit non à la bombe (Montréal, Guérin, coll. " Bibliothèque d'histoire ", 2000) 204 p.
}

\section{Jean-François Auger}

Volume 54, numéro 3, hiver 2001

URI : https://id.erudit.org/iderudit/005317ar

DOI : https://doi.org/10.7202/005317ar

Aller au sommaire du numéro

Éditeur(s)

Institut d'histoire de l'Amérique française

ISSN

0035-2357 (imprimé)

1492-1383 (numérique)

Découvrir la revue

Citer ce compte rendu

Auger, J.-F. (2001). Compte rendu de [OUELLET, Danielle, avec la collaboration de René BUREAU, Franco Rasetti, physicien et naturaliste. Il a dit non à la bombe (Montréal, Guérin, coll. « Bibliothèque d'histoire », 2000) 204 p.] Revue d'histoire de l'Amérique française, 54(3), 475-477.

https://doi.org/10.7202/005317ar d'utilisation que vous pouvez consulter en ligne.

https://apropos.erudit.org/fr/usagers/politique-dutilisation/ 
OUELLET, Danielle, avec la collaboration de René BUREAU, Franco Rasetti, physicien et naturaliste. Il a dit non à la bombe (Montréal, Guérin, coll. «Bibliothèque d'histoire», 2000) 204 p.

$\mathbf{A u} \mathrm{xx}^{\mathrm{e}}$ siècle, la physique fut marquée par les découvertes dans le domaine du nucléaire et par leur application à l'invention d'une arme, la bombe atomique. Le livre de Danielle Ouellet, écrit avec la collaboration de René Bureau, Franco Rasetti, physicien et naturaliste, est la biographie d'un scientifique d'origine italienne qui contribua à ces découvertes et qui, au cours de sa carrière, enseigna la physique à l'Université Laval.

Dans le premier chapitre, nous apprenons que Rasetti s'intéressa à la physique dès le début des années 1920. Avec les Fermi, Amaldi, Segrè et D’Agostino, il participa activement aux travaux du " groupe de Rome» durant plusieurs années. Après des recherches sur l'effet Raman en spectroscopie, il travailla au développement de la physique nucléaire. Il publia d'ailleurs le premier traité sur le sujet en 1936, Il nucleo atomico. Pour mieux situer Rassetti, les auteurs établissent des parallèles entre sa carrière et des événements de l'histoire de la physique. Cependant, la volonté de vulgariser leur sujet les a conduits, parfois, à commettre certaines erreurs. Par exemple, Ernest Rutherford expliqua certes la radioactivité des éléments par la désintégration des atomes; mais il ne réalisa pas de désintégration «artificielle» comme l'affirment les auteurs (p. 28), la première ayant été réalisée par l'Allemand Otto Hahn et l'Autrichienne Lise Meitner en 1939. De plus, rapprocher les travaux du physicien d'origine néo-zélandaise «du rêve séculaire des alchimistes" (loc. cit.) est une exagération typique de la mauvaise vulgarisation et ne contribue en rien à nous faire comprendre la réalité des phénomènes. Finalement, pour assurer une profondeur au contexte historique, il aurait fallu consulter les travaux récents sur le développement de la science dans l'Italie fasciste afin d'identifier les sources primaires pertinentes. Or, les auteurs ne s'en cachent pas, ce chapitre repose uniquement sur des sources secondaires. Ainsi donc, ils n'ont pas 
consulté les archives des institutions scientifiques de Pise, de Rome et de Florence que fréquenta Rasetti.

Dans les deuxième et troisième chapitres, Ouellet et Bureau nous présentent la carrière de Rasetti à l'Université Laval. Pressé de fuir l'Italie fasciste, le physicien se réfugia à Québec en 1939, où lui étaient offerts les postes de professeur et de directeur au département de physique. À plusieurs reprises, il fut sollicité pour collaborer à la conception de la bombe atomique, mais il refusa catégoriquement de s'associer à ce projet militaire. D'ailleurs, dès après que la bombe atomique eut explosé en août 1945, Rasetti entretint une aversion constante pour cette application des connaissances scientifiques. Les valeurs éthiques du scientifique ont justement été retenues par les auteurs comme fil conducteur de la biographie, d'où le sous-titre, Il a dit non à la bombe. Ils désiraient, vraisemblablement, soulever la question de la responsabilité morale du scientifique dans sa pratique. Cependant, cette question est abordée de manière trop superficielle dans l'ouvrage.

À l'aide de l'historiographie des sciences, il est pourtant possible de mieux cerner certaines facettes de la carrière de Rasetti. En premier lieu, l'impact de la Seconde Guerre mondiale sur les pratiques en physique ne semble pas avoir préoccupé les auteurs. Les travaux sur la «migration intellectuelle» leur auraient pourtant permis de préciser le contexte plus vaste dans lequel, en raison de la guerre, se constituèrent les réseaux scientifiques internationaux auxquels participait Rasetti. Même les études historiques sur les recherches ayant mené à la bombe atomique - études pourtant nombreuses - sont ignorées par les auteurs. En second lieu, l'interaction entre l'instrumentation scientifique et les théories physiques fait maintenant partie des interrogations de base des historiens des sciences. Bien entendu, les auteurs ne manquent pas de présenter Rasetti comme un expérimentateur et un concepteur d'instruments hors pair. Au cours de leurs recherches, ils ont consulté les fonds d'archives de l'Université Laval et du Petit Séminaire de Québec; ils ont réalisé des entrevues avec des acteurs de l'époque; et ils disposaient, grâce à Bureau, de documents inédits relatifs à Rasetti. Or les documents grâce auxquels on comprend comment Rasetti mobilisa des ressources financières, forma ses collaborateurs, géra son laboratoire, développa une instrumentation originale et conçut de nouvelles théories physiques n’ont pas été exploités par les auteurs. Au lieu de nous présenter une analyse dynamique de la pratique de la science en laboratoire, analyse à laquelle on s'attendrait, ils se sont plutôt attachés à des détails anecdotiques comme de nous préciser que trois des maisons où habitèrent Rasetti et sa mère à Québec passèrent 
au feu (p. 58) ou, encore, que Rasetti aimait manger des sandwichs sur les pentes de ski du lac Beauport l'hiver... (p. 94)

La partie de la carrière de Rasetti consacrée à la paléontologie et à la botanique est traitée plus longuement, dans le dernier chapitre du livre. Les auteurs ont raison de souligner l'approche multidisciplinaire de ce scientifique. Durant son séjour à Québec, Rasetti renoua avec des pratiques que son père, naturaliste, lui avait transmises à la fin des années 1910. Ainsi découvrit-il des faits nouveaux au sujet des trilobites - crustacés fossiles de l'époque primaire - trouvés dans le conglomérat de Québec, près de Lévis.

À la suite de ces découvertes, l'Université Johns Hopkins lui offrit un poste de professeur de paléontologie, poste qu'il occupa de 1947 à 1967. Rasetti constitua, durant ce temps, des collections de fossiles d'une importance certaine d'un point de vue scientifique; et il écrivit un livre faisant état de plusieurs découvertes dans le domaine de la botanique, $I$ fiori delle Alpi. Or nos auteurs offrent, ici, un simple codicille au sujet d'une partie de la carrière de Rasetti qui, tout compte fait, s'échelonna sur une vingtaine d'années. Ils n'ont pris en compte que la correspondance épisodique de Bureau avec Rasetti ainsi que des entrevues orales et des articles de périodiques d'information générale. Ils n’ont pas considéré l'ensemble des «traces » laissées par le naturaliste aux États-Unis. Cela explique pour une large part l'insuffisance du traitement de cette partie de la carrière du scientifique.

Finalement, il nous faut souligner que le travail de l'éditeur dans le cas de cet ouvrage laisse à désirer. Ce qui peut surprendre d'une collection ("Bibliothèque d’histoire»), qui a réédité ces dernières années les œuvres des Frégault et Séguin. On trouve en effet dans ce livre maintes coquilles. Au lieu de l'information exacte, des points d'interrogation apparaissent dans les bibliographies placées en annexe. Les titres des revues savantes sont citées tantôt en italique, tantôt en caractères gras. Des guillemets d'ouverture annoncent le début d'une citation, sans que des guillemets de fermeture ne viennent en marquer la fin. Plusieurs citations n'ont pas de note ou d'indication de source. Ainsi, le dialogue entre Fermi et Rasetti, dans les rues de Pise en 1919 (p. 1), est impossible à distinguer d'une reconstitution fictive.

En conclusion, il est à souhaiter que paraissent à court terme d'autres études sur Rasetti, le présent ouvrage n'étant malheureusement pas à la hauteur de son sujet ni des avancées récentes de l'histoire des sciences.

JEAN-FRANÇOIS AUGER CRIST et département d'histoire Université du Québec à Montréal 